\section{Perspectivas actuales en la asistencia sanitaria al adulto mayor}

\author{
Ángel Julio Romero Cabrera ${ }^{1}$
}

Forma de citar: Romero Cabrera AJ. Perspectivas actuales en la asistencia sanitaria al adulto mayor. Rev Panam Salud Publica. 2008;24(4):288-94.

Palabras clave: adulto mayor, geriatría, atención a la salud, Cuba.

\footnotetext{
La correspondencia deberá enviarse al doctor Ángel Julio Romero Cabrera, Hospital Universitario "Dr. Gustavo Aldereguía" - Geriatría, Ave. 5 de setiembre esq. 51 A, Cienfuegos, Cienfuegos 55100, Cuba. T.: 53 - 43-52278376. Correo electrónico: jromero@gal.sld.cu
}

Hoy en día resulta cierto que la práctica geriátrica, con su sistema exhaustivo de cuidados a los pacientes más viejos, se está convirtiendo en una poderosa estrategia para los sistemas sanitarios que enfrentan el reto del envejecimiento humano. Este último es un proceso poco comprendido biológicamente, que influye en la expresión clínica de las enfermedades y en la recuperación más torpe de las mismas, pero que por sí mismo no es una enfermedad ni un indicador de deterioro inevitable (1). Por el contrario, la gran mayoría de las personas de edad siguen estando en buenas condiciones físicas hasta bien entradas en la vejez. Además de poder realizar las tareas cotidianas, siguen desempeñando un papel activo en la vida comunitaria. La conservación del máximo nivel de capacidad funcional es tan importante para las personas de edad, como el verse libres de enfermedades (2).

La geriatría ha madurado como especialidad, convirtiéndose en una disciplina de campo amplio, con contenido integral, de acción multidisciplinaria y coordinadora de la asistencia al adulto mayor (AM) desde la gerencia y la organización, hasta el servicio. Sin embargo, está claro que la estrategia actual y futura para enfrentar el reto del envejecimiento no puede ser únicamente la de formar geriatras, lo cual resultaría idílico, sino también la de dotar a las demás especialidades médicas de los atributos y enfoques de la geriatría con una actitud coherente ante la persona de edad avanzada y que el "enfoque geriátrico" se generalice y desarrolle en los servicios, en la docencia y en la investigación (3). Este proceso, denominado "geriatrización" de los servicios permite, a la vez que se va ganando tiempo a la carrera del envejecimiento demográfico, adecuar los recursos materiales y humanos a las necesidades del envejecimiento, lo que resulta más económico en comparación con la formación de nuevos recursos, que consume tiempo y dinero (4).

La geriatrización consiste en ajustar la atención sanitaria que se brinda en los servicios tanto comunitarios como hospitalarios, de acuerdo con las necesidades crecientes de la población que envejece, utilizando las estructuras y tecnología ya instaladas, lo que trae aparejada una gran dosis de capacitación continuada del personal que ahí labora y que incluye a los profesionales, los técnicos y los auxiliares.

Esta estrategia es más barata que la de crear servicios especializados de geriatría - lo que no 
deja de ser importante- con moderna tecnología y equipos multidisciplinarios cuyos integrantes requieren tiempo para su formación (geriatras, licenciados en enfermería, psicólogos, trabajadores sociales, fisioterapeutas, etcétera).

\section{RAZONES PARA LA GERIATRIZACIÓN}

Según Espinosa (3), las razones por las que el estudio y la práctica de los preceptos de la geriatría resultan de sumo interés para la profesión médica y para todos los sectores y las comunidades son varias, a saber: 1) científica o intelectual; 2) demográfica; 3) epidemiológica; 4) económica; 5) sociocultural y 6) personal. En este artículo se hará referencia a cada una de ellas tratando de centrarlas en la perspectiva sanitaria asistencial de los AM.

\section{LA CIENCIA DEL ENVEJECIMIENTO}

A lo largo de la historia el hombre ha estado preocupado por el envejecimiento porque, según decía Cicerón, éste se considera ". . . . una etapa infeliz y está más cerca de la muerte. ..". De ahí que siempre se hayan buscado las causas de este proceso en aras de encontrar su "curación".

Ya en 1903, Michel Elie Metchnikoff, sociólogo y biólogo ruso y Premio Nobel de Medicina y Fisiología, definió la gerontología como la ciencia para el estudio del envejecimiento, con grandes perspectivas para su desarrollo y resultados ulteriores (5). Los gerontólogos caracterizan el envejecimiento por una insuficiencia para mantener la homeostasis bajo condiciones de estrés y esta insuficiencia está asociada con una disminución en la vitalidad y un incremento en la vulnerabilidad del individuo (6).

Se han enunciado múltiples teorías para explicar las causas de este proceso, pero ninguna lo explica por sí sola y muchas, más que las causas, explican las consecuencias o los mecanismos del envejecimiento (7). Lo que sí está firmemente reconocido es que se trata de un proceso irreversible e inevitable y que la extensión de la vida resulta de la interacción entre la dotación genética que se posee y el ambiente en que se vive (8).

El interés científico que despierta el estudio del envejecimiento se ve mermado por las dificultades que presenta el método para abordarlo, entre las que se señalan las siguientes $(6,9)$ :

1. Es un hecho que los AM de la misma edad, varían mucho en cuanto a funcionamiento físico, capacidades, experiencias y comportamiento. La heterogeneidad de las cohortes de ancianos entorpece las investigaciones, y es por eso que se afirma que "los gerontólogos son los únicos científicos que no son capaces de medir aquello sobre lo que trabajan".

2. Resulta difícil diferenciar los cambios producidos por el envejecimiento de aquellos debidos a enfermedades que son fenómenos acompañantes frecuentes, sobre todo los padecimientos crónicos, de la segunda mitad de la vida.

3. Al recurrir a modelos animales, es difícil estudiar los de esperanza de vida larga, pues habría que esperar el tiempo suficiente para que éstos envejezcan. En la práctica, se recurre a animales de vida corta (moscas, helmintos, peces), pero los resultados de los experimentos son menos factibles de extrapolar a los seres humanos.

En opinión del autor del presente trabajo, resultaría más práctico estudiar los factores y mecanismos del envejecimiento saludable y la forma de lograrlo, que el inquirir en las causas específicas de un proceso que hoy se sabe que es continuo, progresivo, complejo y multicausal.

\section{LA RAZÓN DEMOGRÁFICA}

El envejecimiento poblacional es un fenómeno que ocurre en todos los países en mayor o menor grado, lo que provoca un incremento en el número y proporción de las personas ancianas. Baste decir que en 1975 había en el mundo 350 millones de ancianos, cifra que aumentó a 600 millones en 2000 y que casi se volverá a duplicar en 2025 cuando ascienda a 1100 millones (10). Estos cambios demográficos tienen que ver con los avances ocurridos en el siglo XX con la mejoría de las condiciones higiénico-sanitarias y de la nutrición, el control de las enfermedades transmisibles, la disminución de la mortalidad general y de las tasas de mortalidad infantil y, como consecuencia, el aumento de la expectativa de vida de las personas (11). La declinación de la tasa de fertilidad y el control de la natalidad disminuye el número de personas jóvenes y aumenta la proporción de ancianos.

Según el Centro de Estudios de Población y Desarrollo de la Oficina Nacional de Estadísticas (12), Cuba es hoy un país envejecido con $15,8 \%$ de personas de 60 años y más en el total de la población. La disminución de la fecundidad durante las últimas tres décadas es la causa principal del envejecimiento poblacional en Cuba, donde desde hace 28 años la tasa de fecundidad está por debajo del nivel de reemplazo, al quedar menos de una hija por cada mujer en edad reproductiva. El aspecto 
demográfico que más repercute en la asistencia médica es el llamado "envejecimiento del envejecimiento": el segmento de la población de crecimiento más rápido es el de 80 años y más. En los próximos 40 años, el tamaño del grupo de 80 años y más duplicará al de 60 años y más y, si representaba $1 \%$ de la población mundial en 1990, para 2025 llegará a 9\% $(11,13)$. Este grupo es el que demanda mayor atención por problemas de salud, es más dependiente y tiene más problemas sociales y económicos.

\section{EPIDEMIOLOGÍA CAMBIANTE}

La lucha contra el envejecimiento pudiera enfocarse tratando de aumentar el tiempo máximo de vida o la vida promedio. Aumentar el tiempo máximo de vida (115-120 años) puede resultar complicado por limitaciones biológicas evidentes; incrementar el promedio es más sencillo, ya que la prevención es muy útil para garantizarlo (14).

En 1980, Fries (15) conceptualizó el paradigma de la compresión de la morbilidad, donde se postula que la mayoría de las enfermedades son crónicas y ocurren en etapas tardías de la vida, con una gran carga de discapacidad, y que en la medida en que los progresos médicos prolonguen la vida, se espera que haya más años de pobre salud. Si se pospone el comienzo de las enfermedades crónicas, podría reducirse el número de años con discapacidad o (16), lo que es lo mismo, se prolongaría la vida activa de las personas, aproximándola lo más cercana a la muerte. Posponer el comienzo de la morbilidad implica más bien la prevención primaria e intervenciones físicas y conductuales para cambiar los estilos de vida no saludables, y no tanto las expectativas curativas de la ciencia médica tecnólogica actual (17).

Robine y Michel (18) plantean que en el contexto del envejecimiento actual se han introducido cuatro elementos: a) un incremento de las tasas de supervivencia de las personas enfermas, o expansión de la morbilidad; b) un control de la progresión de las enfermedades crónicas, lo cual podría explicar el sutil equilibrio entre la disminución de la mortalidad y el incremento de la discapacidad; c) una mejoría del estado de salud y de las conductas saludables de las nuevas cohortes de AM, o compresión de la morbilidad, y d) la emergencia de poblaciones de personas muy viejas y frágiles (oldest old), lo que podría explicar una nueva expansión de la morbilidad.

A pesar de este mosaico planteado por estos autores, un hecho cierto es que en algunos países como los Estados Unidos de América (EUA) se está comprimiendo la morbilidad, con una disminución de la tasa de discapacidad entre 1 y $2 \%$ anual (15-17).

\section{DE LA FRAGILIDAD A LA DISCAPACIDAD}

El término fragilidad se ha empleado en épocas recientes para hacer referencia a las personas ancianas, teniendo en cuenta su vulnerabilidad y su homeostasis precaria, que las lleva a tener un equilibrio inestable con su medio y semejarse a un cristal, que ante cualquier noxa se rompe (19). El estado frágil es aquel sobre el que es necesario actuar para que el anciano no se discapacite, institucionalice o muera; de aquí que el anciano frágil debe ser el foco, el principal beneficiario de la valoración y asistencia geriátrica.

Cuando no se reconoce ni se toman a tiempo las medidas necesarias para revertirla o detenerla, la fragilidad conduce a la discapacidad. Según Bazlán (20) la secuencia sería la siguiente: la fragilidad provoca disfunción de un órgano o sistema (deterioro funcional); esto limitaría la realización de alguna acción física o mental (limitación funcional), lo que a su vez provocaría dificultad para realizar actividades instrumentadas o básicas de la vida diaria (discapacidad). Algunos autores han definido la fragilidad como una discapacidad preclínica o subclínica, toda vez que ésta puede presentarse en un número significativo de AM que aún no están discapacitados $(21,22)$.

Si desde el punto de vista sanitario y asistencial se quiere lograr un envejecimiento satisfactorio, es necesario prevenir e identificar tempranamente el estado frágil y actuar sobre él, con lo cual se estaría contribuyendo a disminuir la discapacidad y a comprimir la morbilidad.

\section{LOS ESTUDIOS DE MORTALIDAD: ¿QUIÉN APORTA LOS FALLECIDOS?}

La mortalidad constituye un indicador confiable del estado de salud de la población y el estudio de sus causas, y las circunstancias en que ocurre plantean siempre un interés de valor práctico para el médico. En un trabajo (23) realizado en tres provincias cubanas (Ciudad de la Habana, Cienfuegos y Las Tunas) que incluyó el total de fallecidos de 15 años y más, entre 1990 y 1999, se encontró que el grupo de 80 a 84 años fue el que más fallecidos aportó durante todo el decenio, como evidencia de un incremento en el proceso de envejecimiento poblacional en todo el país.

En un trabajo de tesis de especialidad (24) que dirigió el autor del presente trabajo, y donde se estudió la mortalidad de los AM (60 años y 
más) en el decenio 1990-1999 en la provincia de Cienfuegos, se encontró que las primeras cinco causas de muerte correspondieron a: enfermedades del corazón, tumores malignos, enfermedades cerebrovasculares, influenza y neumonía y accidentes. Como puede verse, cuatro eran padecimientos crónicos no transmisibles, y todos azote de la ancianidad.

Todo lo expuesto hasta aquí lleva a afirmar que existe una línea continua en el discurso entre envejecimiento y muerte: envejecimiento individual y demográfico, envejecimiento del envejecimiento, morbilidad, fragilidad, discapacidad y mortalidad.

\section{LOS RECURSOS Y LOS COSTOS}

La carga que el envejecimiento demográfico impone a la sociedad se refleja en términos económicos. Es indiscutible que el aumento de enfermedades crónicas a medida que avanza la vida, unido a condicionantes psicosociales específicas, hacen que el grupo poblacional más viejo necesite y consuma más recursos en el orden de: medicamentos, atención médica y de enfermería, rehabilitación, asistencia social, instituciones para ancianos, etcétera (25).

Según Duthie (26) los ancianos consumen $30 \%$ de los servicios que prestan los internistas, $24 \%$ de las visitas a consulta, $25 \%$ del consumo de medicamentos y $25 \%$ de los gastos totales de salud.

En los EUA, el incremento en la atención médica del anciano desde 1960 hasta el 2000 se asoció con una elevación de los costos por año de vida ganado en la expectativa de vida. Este promedio fue de unos \$31 600 a los 15 años de edad, \$53 700 a los 45 años y $\$ 84700$ a los 65 años, edad en que los costos crecieron más rápido que la expectativa de vida: el costo por año de vida ganado fue de $\$ 121000$ entre 1980 y 1990 y de $\$ 145000$ entre 1990 y 2000 (27).

Dos ejemplos de enfermedades crónicas que gravitan extraordinariamente sobre los costos son las fracturas y la insuficiencia cardiaca. Unas 310000 fracturas ocurren cada año en la población anciana en el Reino Unido. El costo del cuidado y apoyo social para estos pacientes es de alrededor de 2,4 billones de euros (28). La insuficiencia cardiaca representa una enorme carga, al consumir $2 \%$ de todos los gastos de salud en los EUA (29). Se ha reconocido la asociación entre la pobreza y el deterioro de la salud de los ancianos, y está perfectamente demostrado que las limitaciones funcionales entre 55 y 84 años están inversamente relacionadas con la clase social y el gradiente socioeconómico (30).

\section{LA RAZÓN SOCIOCULTURAL}

Se ha dicho, con razón, que "se envejece según se ha vivido" y esto presupone que los cambios en la esfera social cuando el individuo llega a una edad avanzada, están determinados por su conducta social previa (31). La posición del individuo en la sociedad se modifica en el curso de su vida y depende de factores biológicos, de patrones de conducta, de su personalidad, del rol social desempeñado y, en gran medida, del sistema social y el momento histórico en que se desarrolla (32).

La sociedad actual no debe "medicalizar" los problemas de las personas de edad y le conviene adoptar una estrategia multisectorial y comunitaria donde el propio AM, con sus experiencias y motivaciones, sea el principal protagonista. La conservación de las relaciones intergeneracionales es crucial para la prosperidad de las edades avanzadas $y$, para ello, las generaciones pregeriátricas deben actuar en dos sentidos: primero, reconociendo los valores de la vejez, reforzando su imagen y nutriéndose de su experiencia y, segundo, preparándose para la vejez, pues la mayoría de sus miembros llegarán a esta etapa de la vida.

La sociedad debe estructurarse sobre la base de valores humanos enaltecedores del hombre como ser social $\mathrm{y}$, aunque preocupen los efectos económicos del envejecimiento, no debe dejarse de prestar atención a la ancianidad, estableciendo programas integrales para ellos, que tanto dieron en su juventud en la construcción de los valores que disfruta la sociedad actual.

Es vital crear una cultura para el envejecimiento (gerocultura) propiciadora de mecanismos reflexivos, que permita una dinámica favorable en los planos estatal, comunitario y familiar, hasta llegar al individuo, no sólo sobre el envejecimiento en sí, sino desde el envejecimiento. La participación individual, familiar y comunitaria en el cambio de óptica y actitud que se impone, precisa un reconocimiento de las potencialidades del AM; el reforzamiento de su imagen de memoria viva, de transmisor del patrimonio cultural, así como de su inserción y participación social en este proceso de transmisión, constituye la base de la gerocultura (33). Este proceso no puede ser impuesto desde fuera y debe contar con la voluntad y motivaciones de los AM, quienes de por sí juegan un papel protagónico.

\section{OBJETIVO, METAS Y MÉTODO DE LA ASISTENCIA GERIÁTRICA}

La práctica geriátrica no es fragmentada ni subespecializada. Es un sistema exhaustivo de cuidados de los pacientes ancianos, que incluye los prin- 
cipios de la medicina del adulto, modifica estos principios y los acomoda a los cambios asociados al envejecimiento, empleando por lo general una estrategia multidisciplinaria, un abordaje en equipo.

El objetivo de la asistencia al AM está centrado en una atención integral y continuada de su salud que va desde la promoción, la prevención, el diagnóstico, el tratamiento y la rehabilitación, con el propósito universal de mejorar su calidad de vida, término que, en el caso de las personas mayores, está íntimamente relacionado con su capacidad funcional $(3,34)$.

El objetivo principal de los programas de prevención en los ancianos debe ser tratar de mantener y mejorar su calidad de vida previniendo la aparición de enfermedades físicas y mentales y sus secuelas, manteniendo un grado de autonomía tal que les permita la integración social en su medio. La meta es prevenir la declinación, la fragilidad y la dependencia evitable $(35,36)$.

Según Guillén (37), el anciano necesita más cuidados que tratamiento (care vs. cure); necesita un plan de cuidados integral que incluye no sólo los aspectos biomédicos específicos de las enfermedades que pueda presentar, sino también elementos tan importantes como los factores psicológicos, sociales y económicos que repercuten en su capacidad funcional y minan su calidad de vida. La estructuración de un plan de cuidados racional, coherente y eficaz donde se valoran riesgos y beneficios requiere la integración y el concurso de varias disciplinas con un abordaje multidisciplinario, en equipo, o interdisciplinario para resolver estos problemas. Los componentes básicos de un equipo de atención geriátrica comprenden al geriatra (o en su defecto al internista o especialista en medicina general integral) así como a los licenciados en enfermería, el psicólogo y el trabajador social, con el apoyo de otros profesionales y técnicos entre los que están el fisioterapeuta, el ergoterapeuta, el podólogo, el nutricionista, el estomatólogo, etcétera.

La función y utilidad de los servicios geriátricos especializados quedan bien documentadas al satisfacer las necesidades del anciano frágil, objeto principal de su atención. Estos servicios desempeñan un papel muy importante a través de la consulta y la formación, influyendo en el resto de los servicios de atención sanitaria para que lleguen a sensibilizarse con el manejo de los problemas de los $\operatorname{AM}(38,39)$.

El método que constituye la piedra angular para conseguir estos propósitos es la valoración geriátrica, definida como un proceso diagnóstico multidimensional, encaminado a determinar las capacidades y problemas biomédicos, psicológicos, socioeconómicos y funcionales del AM con el objetivo de desarrollar un plan general de tratamiento y seguimiento de largo plazo (40). Es conveniente insistir en que el componente funcional de la evaluación es la medida resumen de las capacidades, de la independencia y del estado de salud general del anciano.

La evaluación geriátrica es un método de valoración integral que comprende, en los aspectos biomédicos: los diagnósticos presentes y pasados (historia clínica tradicional), y la nutrición y la medicación; entre los aspectos psicológicos: la función cognitiva (funciones mentales superiores) y la función afectiva (ansiedad, depresión); entre los aspectos sociales: la convivencia, el estado de la vivienda, el apoyo social y el nivel socioeconómico, y entre los aspectos funcionales: el grado de independencia para la realización de las actividades de la vida diaria básicas (alimentarse, bañarse, vestirse, uso de retrete, movilidad y continencia de esfínteres) e instrumentales (uso de transporte, compras, manejar dinero, telefonear, realizar tareas domésticas como cocinar o lavar y autoadministrarse los medicamentos). El listado de problemas que emana de estos cuatro aspectos constituye la base para elaborar un plan de cuidados encaminado a su solución y debe ser individualizado para cada AM.

En 1984, Rubenstein y cols. (41) notificaron que los ancianos evaluados y tratados en unidades especializadas tenían una menor tasa de ingreso a hogares de ancianos y una reducción de $50 \%$ de mortalidad al año, que los evaluados según métodos tradicionales. Hoy se sabe que el poder de la geriatría no es tan dramático al quedar demostrado, en un ensayo aleatorio (42) que incluyó 11 centros y 1388 ancianos, que aquellos que recibieron un cuidado geriátrico especializado mejoraron más en la función y en la calidad de vida que los que recibieron un cuidado usual, aunque con costos y mortalidad similares. Estudios recientes (43) también notifican beneficios con la aplicación de la valoración geriátrica exhaustiva en el AM en la comunidad, con disminución de la discapacidad y del riesgo de hospitalización.

Los ancianos que parecen obtener mayor beneficio son los mayores de 75 años, los que tienen discapacidades leves o moderadas, posiblemente en riesgo de ser emplazados en un hogar de ancianos y los que tienen un pobre entorno social; la mayoría de todos ellos cae dentro de la categoría de anciano frágil.

Respecto a la valoración geriátrica, el autor del presente trabajo comparte la opinión de Perlado (44), quien señala que valorar significa comprender y no sencillamente aplicar escalas y reglas de medición complejas y sofisticadas sin interpretar los diferentes matices de la situación ni apreciar el conjunto, sabiendo que éste, en lo que al AM respecta, no siempre es la suma de las partes. 
Finalmente, una meta que no puede quedar olvidada en la asistencia a los ancianos es la de proveer de cuidados cuando no hay curación, en aquellas enfermedades o problemas que tienen un pronóstico ominoso y que han sido conceptualizados como cuidados terminales o, con más propiedad, cuidados al final de la vida. Un aspecto importante de estos cuidados es el enfoque en el manejo sintomático para aportar comodidad, dignidad y calidad de vida (45).

Según Jiménez Rojas (46), los cuidados al final de la vida forman parte de los objetivos de la medicina y su contenido fundamental comprende el control sintomático, la importancia del respeto al paciente y la necesidad de un marco ético en la toma de decisiones.

Dado que el personal que atiende ancianos tendrá que enfrentar en algún momento el problema de la muerte, resulta más adecuado considerar en la práctica clínica la adopción de actitudes paliativas en el plan terapéutico global, que promover los cuidados paliativos como una actividad terapéutica aislada en el curso de una enfermedad incurable.

\section{RAZÓN PERSONAL}

Una frase de Lin Yutang podría ilustrar por qué, personalmente, cabe preocuparse, a la vez que ocuparse, de las acciones para lograr un envejecimiento satisfactorio. Él decía: "Ninguno puede realmente detener el envejecimiento. Y como es inútil luchar contra la naturaleza, se podría al menos envejecer graciosamente. La sinfonía de la vida debería acabar con un gran final de paz y serenidad, de bienestar material y de contenido espiritual, y no con el estallido de un tambor roto o de címbalos quebrados", a lo que cabe agregar: no olvidemos que los ancianos del mañana somos nosotros mismos.

\section{SYNOPSIS}

\section{Current perspectives on health care for the older adult}

Standing up to the challenges of aging requires a coordinated strategy, disseminating a geriatric focus among all the specialty areas that serve the older adult. This article addresses the reasons that justify this focus and covers the objectives, goals, and methods currently being proposed to develop the approach. Preventive and curative actions, but most importantly, the overall care of seniors and improvements to their functional status, are priorities for securing a better quality of life in old age.

Key words: aged, geriatrics, health care, Cuba.

\section{REFERENCIAS}

1. Campion MD. Specialized care for elderly patients N Engl J Med. 2002; 346(12):874.

2. Organización Panamericana de la Salud. El envejecimiento: cómo superar mitos. Washington DC: OPS;1999.

3. Espinosa Brito A. La geriatría: ¿una especialidad? En: Espinosa A, Romero AJ, eds. Temas de gerontogeriatría. Cienfuegos: Editorial Finlay; 1990. Pp.1-10.

4. Tamayo S. ¿Geriatrización de los servicios de salud? Gerosur 1997;1(1):1-2.

5. Prieto O. Gerontología y geriatría. Breve resumen histórico. RESUMED 1999; 12(2):51-4.

6. Zarzosa López MC, Castro Carratalá E. ¿Sabemos qué significa envejecer? Geriátrika 2006;22(2):56-9.

7. Ruiz Torres A. Envejecimiento: causa, mecanismos y regulación. Rev Esp Geriatr Gerontol. 2001;36(85):13-9.

8. Westerndorp RGJ. What is healthy aging in the 21th century? Am J Clin Nut. 2006;83(2):404-9.
9. Rodriguez Mañas L. Biología del envejecimiento. En: Guillén F, Pérez del Molino J, eds. Síndromes y cuidados en el paciente geriátrico. Barcelona: Masson S.A.; 1994. Pp. 11-6.

10. Oficina Nacional de Estadísticas. El envejecimiento de la población: Cuba y sus territorios. Cifras e indicadores para su estudio. Centro de Estudios de Población y Desarrollo. La Habana: Oficina Nacional de Estadísticas; 2001.

11. Restrepo HE, Rozental M. The social impact of aging populations: some major issues. Soc Aci Med. 1994;39(9):1322-38.

12. Peláez O. Disminución acelerada de la natalidad y envejecimiento poblacional. Reto inaplazable para los cubanos. [Sitio en Internet] Diario Granma. Hallado en: http://www.granma.cubaweb.cu. Acceso el 30 octubre 2006.

13. Cassel CK. Successful aging: How increased life expectancy and medical advances are changing geriatric care. Geriatrics. 2001;56:35-9.
14. Quintana O. Los objetivos de la medicina. Rev Calidad Asist. 2003;10(2): $132-5$.

15. Fries JF. Aging, natural death and the compression of morbidity. N Engl J Med. 1980;303:130-5.

16. Fries JF. Measuring and monitoring success in compressing morbidity. Ann Intern Med. 2003;139:455-9.

17. Fries JF. Successful aging - an emerging paradigm of gerontology. Clin Geriatr Med. 2002;18:371-82.

18. Robine JM, Michel JP. Looking forward to a general theory on population aging. J Gerontol A Biol Sci Med Sci. 2004;59: 590-7.

19. Romero Cabrera AJ. La fragilidad como punto de corte en geriatría. Geriátrika 2006;22(2):42-6.

20. Bazlán JJ, González-Montalvo JI, Solano JJ, Hornillos M. Atención sanitaria al anciano frágil: de la teoría a la evidencia científica. Med Clin (Barc). 2000;11: 704-17. 
21. Lluis Ramos GE, Llibre Rodríguez JJ. Fragilidad en el adulto mayor: un acercamiento. Rev Cubana Med Gen Integr [publicación periódica en línea] 2004 [citada 2007 jun 12]; 20(4):[10 pantallas]. Hallado en: URL: http://scielo.sld.cu/ scielo.php?script $=$ sci_arttext\&pid $=$ S086421252004000400009\&lng=es\&nrm = iso\&tlng=es

22. Fried LP, Guralnik JM. Disability in older adults: evidence regarding significance, etiology, and risk. J Am Geriatr Soc. 1997:45:92-100.

23. Espinosa A, Quintero Y, Cutiño Y, Romero AJ, Bernal JL. Mortalidad del adulto en tres provincias cubanas (19901999). En: Íñiguez L, Pérez OE, eds. Heterogeneidad social en la Cuba actual. Centros de estudios de salud y bienestar humano. La Habana: Universidad de La Habana; 2004. Pp. 163-93.

24. Estévez Téllez M. Mortalidad geriátrica en el decenio 1990-99. Provincia de Cienfuegos [tesis]. Cienfuegos: Hospital Universitario “Dr. Gustavo Aldereguía Lima"; 2001.

25. Guralnik JM, Alecxih L, Branch LG, Wiener JM. Medical and long-term care costs when older persons become more dependents. Am J Pub Health. 2002; 92(8):1244-5.

26. Duthie ED. Geriatrics and geriatricians. En: Duthie EH. Practice of geriatrics. 2a ed. Philadelphia: WB Saunders Co; 1998. Pp. 3-5.

27. Cutler $\mathrm{DM}$, Rosen $\mathrm{AB}$, Vijan $\mathrm{S}$. The value of medical spending in the United States, 1960-2000. N Engl J Med. 2006; 355(9):920-7.

28. Woolf AD, Akesson K. Preventing fractures in elderly people. BMJ. 2003;327: 89-95.
29. McMurray JJV, Pfeffer MA. Heart failure. Lancet. 2005;365:1877-89.

30. Minkler M, Fuller-Thomson E, Guralnik JM. Gradient of disability across the socioeconomic spectrum in the United States. N Engl J Med. 2006;355:695-703.

31. Romero Cabrera AJ. Mirando la vejez a través del cristal sociológico. Geriatrianet.com [publicación periódica en línea] 2006 [citada 2007 dic 6]; 8(1): [13 pantallas]. Hallado en: URL: http://www. geriatrianet.com/index.php/journal/ article/view $/ 51 / 83$

32. Dahm I, Gussler A, Huttner H, Huttner I, Klemm P, Kunzendorf E, et al. Sociología para médicos. La Habana: Editorial Ciencias Sociales; 1990. Pp. 117-20.

33. González Braniella MA. La dimensión cultural del desarrollo y la vejez. En: Sansó F, ed. Salud familiar y desarrollo sociocultural. La Habana: Bibliografía Básica Maestría Longevidad Satisfactoria; 2005. Pp. 50-2.

34. González Guerrero JR, Jaramillo E Ansón M. Medicina preventiva en las personas mayores. Tema monográfico La salud del anciano. [Sitio en Internet] Ediciones Doyma. Hallado en: http:/ / www. doyma. es. Acceso el 8 febrero 2006.

35. Prado F, Petidier R. Promoción de salud y medicina preventiva en el anciano. En: Rodriguez Mañas L, Solano JJ, eds. Bases de la atención sanitaria al anciano. Madrid: SEMEG; 2001. Pp. 83-124.

36. Mehr DR, Tatum PE. Primary prevention of diseases of old age. Clin Geriatr Med. 2002;18:407-30

37. Guillén Llera F. Patología del envejecimiento. En: Guillén F, Pérez del Molino J, eds. Síndromes y cuidados en el paciente geriátrico. Barcelona: Masson S.A.; 1994. Pp. 37-46.
38. Fisher RH. Caídas en la persona mayor y el papel de la geriatría. Rev Esp Geriatr Gerontol. 2003;38(2):7-99.

39. Fisher RH. The role of specialized geriatric services in acute hospitals. Geriatrics and Ageing [publicación periódica en línea] 2002 [citada 2007 jul 6]; 5: [4 pantallas]. Hallado en: URL: http://www.geriatricsandaging. com

40. Vega E. Evaluación del anciano. En Prieto O, Vega E, eds. Temas de gerontología. La Habana: Editorial Científico Técnica; 1996. Pp. 119-25.

41. Rubenstein LZ, Josephson KR, Wieland GD, English PA, Sayre JA, Kane RL. Effectiveness of a geriatric evaluation unit. A randomized clinical trial. N Engl J Med. 1984;311:1664-70.

42. Cohen HJ, Feussner JR, Weinberger $M$ Carnes M, Hamdy RC, Hsieh F, et al. A controlled trial of inpatient and outpatient geriatric evaluation and management. N Engl J Med. 2002;346(12): 905-12.

43. Stott DJ, Buttery AK, Bowman A, Agnew R, Burrow K, Mitchell SL, et al. Comprehensive geriatric assessment and home-based rehabilitation for elderly people with a history of recurrent non-elective hospital admissions. Age Ageing. 2006;35:487-91.

44. Perlado F. Valoración geriátrica. Rev Esp Geriatr Gerontol. 2001;36(supl 5): 25-31.

45. Romero Cabrera AJ, Nieto Cabrera R. Marco teórico de los cuidados terminales en el paciente geriátrico. Geriátrika 2004;20(5):198-205.

46. Jiménez Rojas C. Cuidados al final de la vida. Rev Esp Geriatr Gerontol. 2002; 37(5):241-3.

\section{Mantenerse en forma para la vida. Necesidades nutricionales de los adultos mayores}

La Organización Panamericana de la Salud (OPS) reconoce que proteger la salud de los adultos mayores es uno de los principales desafíos que enfrenta la salud pública en el siglo XXI. Una buena alimentación y el mantenerse en forma son maneras de garantizar la salud y el bienestar en la vejez. En este marco, la OPS se complace en presentar su más reciente publicación, Mantenerse en forma para la vida: necesidades nutricionales de los adultos mayores, obra que representa una fuente autorizada de información sobre la alimentación y actividad física recomendadas para los adultos mayores.
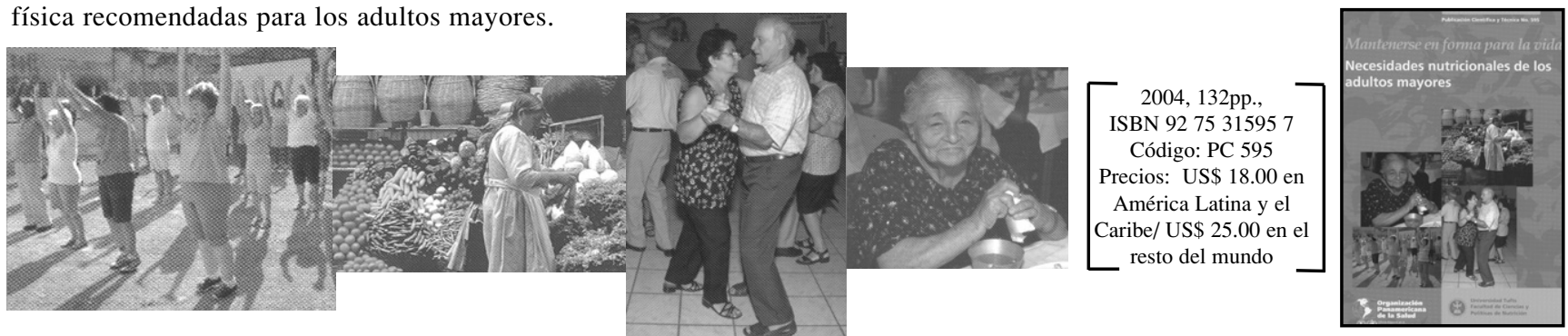

Esta publicación será de mucha utilidad para nutricionistas, médicos generales, gerontólogos, personal de enfermería, proveedores de atención, formuladores de políticas públicas, salubristas y trabajadores sociales.

Adquiera esta publicación por medio de la librería en línea de la OPS: http://publications.paho.org; correo electrónico: paho@ brightkey.net 